\title{
Índices ecocardiográficos y estancia hospitalaria en pacientes con insuficiencia cardiaca aguda en el Hospital Guillermo Almenara Irigoyen, 2015
}

\author{
Ciro Barrantes Alarcón* 1,a
}

\author{
RESUMEN
}

Objetivo: Conocer la asociación entre el tiempo de estancia hospitalaria en casos de insuficiencia cardiaca aguda con cuatro índices ecocardiográficos en los pacientes hospitalizados en la Unidad de Cuidados Coronarios del Hospital Guillermo Almenara. Los índices estudiados son la relación velocidad de onda E por doppler pulsado del flujo mitral/ velocidad de onda e' por doppler tisular en anillo mitral (E/e'), el diámetro de la vena cava inferior, la presión sistólica de la arteria pulmonar y el índice de colapso de la vena cava inferior.

Materiales y métodos: Se realizó un estudio cuantitativo, descriptivo, retrospectivo y correlacional con todos los pacientes hospitalizados por insuficiencia cardiaca aguda durante el año 2015. La asociación entre el tiempo de estancia hospitalaria y la relación E/e', el diámetro de vena cava inferior y la presión sistólica de la arteria pulmonar se estudió con la prueba de Pearson. Se empleó odds ratio (OR) para establecer la relación de la estancia hospitalaria con el índice de colapso de vena cava inferior.

Resultados: Se incluyeron 65 pacientes con una media de edad de 67,8 años y la mayoría era de sexo masculino (61,5 \%). La media de la estancia hospitalaria fue 5,83 días. El diagnóstico previo de insuficiencia cardiaca mostró asociación con una estancia hospitalaria mayor a 5 días (prueba de la $\mathrm{X}^{2}$ ). No hubo asociación entre la fracción de eyección del ventrículo izquierdo y la estancia hospitalaria (OR de 1,016 [IC $95 \%=0,380-2,717]$ ). Se encontró asociación significativa entre el incremento en la relación E/e' $(p=0,002)$, el mayor diámetro de vena cava inferior $(p=0,006)$ y el índice de colapso de vena cava inferior menor a $50 \%(p<0,0000)$ con la mayor estancia hospitalaria.

Conclusiones: El incremento de la relación E/e', el mayor diámetro de la vena cava inferior y el índice de colapso de la vena cava inferior menor a 50 \% están asociados a una estancia hospitalaria prolongada en pacientes con insuficiencia cardiaca aguda en una unidad de cuidados coronarios.

Palabras clave: Insuficiencia cardiaca; Ecocardiografía; Hospitalización (Fuente: DeCS BIREME).

\section{Echocardiographic indices and hospital stay among patients with acute heart failure at the Hospital Guillermo Almenara Irigoyen, 2015}

\begin{abstract}
Objective: To know the association between the length of hospital stay for acute heart failure and four echocardiographic indices among inpatients in the Coronary Care Unit of the Hospital Guillermo Almenara Irigoyen. The study indices were the ratio of mitral valve inflow velocity of pulsed-wave Doppler E and mitral annular tissue velocity of pulsed-wave Doppler e' (E/e'), inferior vena cava diameter, inferior vena cava collapse rate and pulmonary artery systolic pressure. Materials and methods: A quantitative, descriptive, retrospective and correlational study was conducted with all patients hospitalized for acute heart failure during 2015. The association that the length of hospital stay has with the E/e' ratio, inferior vena cava diameter and pulmonary artery systolic pressure was analyzed using Pearson's correlation coefficient. The relationship between the length of hospital stay and the inferior vena cava collapse rate was determined using the OR. Results: The study population consisted of 65 patients whose mean age was 67.8 years and who were mainly males $(61.5 \%)$. The mean hospital stay was 5.83 days. The diagnosis of heart failure was associated with a hospital stay greater than 5 days (chi-square test). There was no association between left ventricular ejection fraction and hospital stay $(O R=1.016 ; 95 \% \mathrm{Cl}=0.380-2.717)$. A significant association was found between a longer hospital stay and the increase in the E/e' ratio $(p=0.002)$, larger inferior vena cava diameters $(p=0.006)$ and inferior vena cava collapse rates less than $50 \%(p<0.0000)$.

Conclusions: The increase in the E/e' ratio, the larger inferior vena cava diameter and the inferior vena cava collapse rate less than $50 \%$ are associated with a long hospital stay among inpatients with acute heart failure in a coronary care unit.
\end{abstract}

Keywords: Heart failure; Echocardiography; Hospitalization (Source: MeSH NLM).

1 Médico Cardiólogo.

a Hospital Nacional Guillermo Almenara. Lima, Perú.

*Autor corresponsal.

https://doi.org/10.24265/horizmed.2020.v20n3.02 


\section{INTRODUCCIÓN}

La insuficiencia cardiaca aguda es una enfermedad de alta prevalencia y una de las causas más frecuentes de hospitalización en los servicios de cardiología, medicina y cuidados intensivos en el mundo ${ }^{(1)}$. Existe un aumento en su prevalencia relacionado a una mayor expectativa de vida de la población (2). La insuficiencia cardiaca aguda se define como el deterioro rápido y progresivo de la función del corazón o como la aparición de nuevos síntomas y signos de insuficiencia cardiaca que necesitan un tratamiento inmediato y que ocasionan una hospitalización ${ }^{(3)}$.

Durante un evento de insuficiencia cardiaca las presiones intracardiacas se incrementan, esto origina cambios estructurales y funcionales en el corazón que pueden ser determinados por ecocardiografía y están asociados a pronósticos adversos como mortalidad luego del alta o nuevas hospitalizaciones ${ }^{(3,4)}$. Los índices ecocardiográficos que cambian son la relación E/e' (en los casos con fracción de eyección preservada o disminuida) ${ }^{(5-9)}$, el diámetro e índice de colapso de vena cava inferior $(9,10)$ y la presión sistólica de arteria pulmonar (11-15). Sin embargo, en un episodio agudo, la importancia pronóstica de estos índices no está bien establecida. La fracción de eyección del ventrículo izquierdo se asocia a mortalidad, pero no a estancia hospitalaria ${ }^{(3,16)}$.

Los pacientes con insuficiencia cardiaca se hospitalizan en una unidad coronaria durante cinco días, en promedio ${ }^{(1)}$. Los periodos más extensos a esa media se consideran estancias prolongadas y ocurren por una inadecuada respuesta al tratamiento o por mayor gravedad.

La relación E/e' se considera elevada cuando es mayor a $13^{(17)}$, este valor se obtiene con el promedio de septal y lateral o el que técnicamente pueda medirse ${ }^{(18)}$. En relación a la vena cava inferior, su diámetro esta aumentado si es mayor a $21 \mathrm{~mm}{ }^{(19)}$, y el índice de colapso es anómalo si es menor a $50 \%{ }^{(20)}$. La presión sistólica de la arteria pulmonar es anormal cuando es mayor a $35 \mathrm{mmHg}{ }^{(18-20)}$.

El objetivo de este trabajo fue conocer la relación entre los índices ecocardiográficos E/e', el diámetro de la vena cava inferior, el índice de colapso de la vena cava inferior y la presión sistólica de la arteria pulmonar con el tiempo de estancia hospitalaria en pacientes con insuficiencia cardiaca aguda internados en la Unidad de Cuidados Coronarios Hospital Guillermo Almenara en 2015.

\section{MATERIALES Y MÉTODOS}

\section{Diseño y población de estudio}

La presente investigación fue de tipo cuantitativo y de diseño observacional, descriptivo, transversal, correlacional y retrospectivo.
La población del estudio estuvo conformada por todos los pacientes con diagnóstico de insuficiencia cardiaca aguda, según la guía de la Sociedad Europea de Cardiología (21), mayores de 18 años, hospitalizados en la unidad coronaria del Hospital Nacional Guillermo Almenara Irigoyen (HNGAI) durante el año 2015. Los criterios de inclusión fueron tener un diagnóstico realizado por un cardiólogo clínico del Servicio de Cardiología del HNGAl y estar hospitalizado por más de 48 horas. Se excluyeron a los pacientes fallecidos en las primeras 48 horas de hospitalización en la Unidad de Cuidados Coronarios, las gestantes, los casos complicados (con infecciones, enfermedad renal descompensada, enfermedad cerebrovascular o infarto agudo de miocardio), las enfermedades cardiacas infiltrativas y las que presentan algún grado de disfunción diastólica , las cardiomiopatías (hipertróficas y restrictivas), la pericarditis constrictiva, cor pulmonale, una mala ventana acústica que impida una adecuada evaluación, portar marcapasos y tener una prótesis valvular mitral.

\section{Variables y mediciones}

La información obtenida de la historia clínica se registró en una ficha de recolección de datos diseñada para esta investigación. Para determinar si las características de cada paciente al momento del ingreso se relacionaban con una mayor estancia hospitalaria, la escala cuantitativa de estancia hospitalaria se convirtió en una variable cualitativa ordinal, que tomó como punto de corte la mediana y media obtenidas para la variable estancia hospitalaria o días en unidad de cuidados coronarios (UCC). Esta variable ordinal de estancia hospitalaria se comparó con la presencia o ausencia de signos, síntomas y antecedentes clínicos buscando una asociación entre las variables con la prueba de la $X^{2}$.

\section{Análisis estadístico}

Empleamos odds ratio (OR) para determinar si existe asociación entre fracción de eyección ventricular izquierda y estancia hospitalaria. Ambas variables se convirtieron en variables cualitativas ordinales con dos categorías cada una: la fracción de eyección ventricular izquierda (categorías: menor a $40 \%$ y mayor/igual a $40 \%$ ) y la estancia hospitalaria (categorías: hasta cinco días y mayor a cinco días).

La asociación entre las variables principales del estudio (E/e', diámetro de vena cava inferior y presión sistólica de arteria pulmonar) con estancia hospitalaria se analizó con la prueba de Pearson. El índice de colapso de la vena cava inferior $\mathrm{VCl}-\mathrm{IC}$ es una variable dicotómica (menor a $50 \%$ y mayor a $50 \%$ ), por lo que se utilizó la prueba de la $X^{2}$ para establecer su asociación con la estancia hospitalaria. Si se determina que existió asociación entre las variables estudiadas, se establecería un modelo predictivo de comportamiento entre ellas a través de un análisis de regresión lineal. 


\section{Consideraciones éticas}

Este estudio es retrospectivo observacional por lo que se revisaron historias clínicas. Para ello, se obtuvo el permiso del Comité de Ética del Hospital Guillermo Almenara; y se guardó la confidencialidad de los nombres de los pacientes.

\section{RESULTADOS}

En el 2015 ingresaron al HNGAl 360 pacientes con el diagnóstico de insuficiencia cardiaca aguda De ese grupo, 96 se hospitalizaron en la Unidad de Cuidados Coronarios y 65 de ellos cumplieron los criterios de inclusión. La edad media fue de 67,8 años. Todos los pacientes ingresaron con el diagnóstico de insuficiencia cardiaca aguda. Las características clínicas más frecuentes fueron disnea $(96,90 \%)$ y crepitantes $(83,10 \%)$. En la radiografía torácica, las alteraciones más frecuentes fueron la cardiomegalia $(76,90 \%)$ y la congestión pulmonar $(75,40 \%)$. El antecedente más frecuente fue la hipertensión arterial $(63,10 \%)$.

La media de la estancia hospitalaria fue 5,83 días (Figura 1). Se realizó un análisis cualitativo con la prueba de la $X^{2}$ y se encontró que la variable diagnóstico previo de insuficiencia cardiaca fue la única que mostró asociación con una estancia hospitalaria mayor a 5 días (OR de 4,16; $p=0,016)$. Los resultados se muestran en la tabla 1.

Tabla 1. Análisis de asociación cualitativa entre variables clínicas y una estancia mayor a 5 días. Hospital Guillermo Almenara 2015

\begin{tabular}{|c|c|c|c|c|c|c|}
\hline Variables clínicas & $\begin{array}{l}\text { Mayor } \\
5 \text { dias } \\
(n=30)\end{array}$ & $\begin{array}{l}\text { Menor o } \\
\text { igual } 5 \text { dias } \\
(n=34)\end{array}$ & OR & $\begin{array}{l}\text { IC } \\
\text { Límite } \\
\text { inferior }\end{array}$ & $\begin{array}{l}\text { Límite } \\
\text { superior }\end{array}$ & $p$ \\
\hline Disnea & 30 & 32 & 4,69 & 0,21 & 101,72 & 0,280 \\
\hline Dolor torácico & 11 & 15 & 0,74 & 0,27 & 2,00 & 0,553 \\
\hline Palpitaciones & 4 & 7 & 0,62 & 0,16 & 2,27 & 0,467 \\
\hline Hipertensión arterial (HTA) & 16 & 24 & 0,48 & 0,17 & 1,33 & 0,160 \\
\hline Diabetes mellitus (DM) & 7 & 8 & 0,99 & 0,31 & 3,15 & 0,985 \\
\hline Válvula mitral & 6 & 6 & 1,17 & 0,33 & 4,10 & 0,810 \\
\hline Válvula aórtica & 7 & 5 & 1,77 & 0,50 & 6,29 & 0,378 \\
\hline Infarto previo & 9 & 13 & 0,69 & 0,24 & 1,96 & 0,489 \\
\hline Presencia de soplos & 18 & 15 & 1,90 & 0,70 & 5,14 & 0,157 \\
\hline Presencia de edemas & 18 & 17 & 1,50 & 0,56 & 4,05 & 0,423 \\
\hline Ingurgitación yugular & 13 & 15 & 0,97 & 0,36 & 2,61 & 0,950 \\
\hline Crepitantes & 26 & 27 & 1,69 & 0,44 & 6,44 & 0,443 \\
\hline Fibrilación auricular & 12 & 15 & 0,84 & 0,31 & 2,29 & 0,739 \\
\hline Hipertrofia ventricular & 10 & 12 & 0,92 & 0,33 & 2,58 & 0,869 \\
\hline Crecimiento de ventrículo derecho & 5 & 2 & 3,20 & 0,57 & 17,89 & 0,168 \\
\hline Crecimiento de aurícula izquierda & 4 & 8 & 0,50 & 0,13 & 1,87 & 0,297 \\
\hline Alteraciones ST, T & 9 & 12 & 0,79 & 0,27 & 2,25 & 0,653 \\
\hline Necrosis & 1 & 4 & 0,26 & 0,03 & 2,45 & 0,210 \\
\hline Diagnóstico previo de ICC & 26 & 20 & 4,16 & 1,24 & 13,89 & 0,016 \\
\hline
\end{tabular}




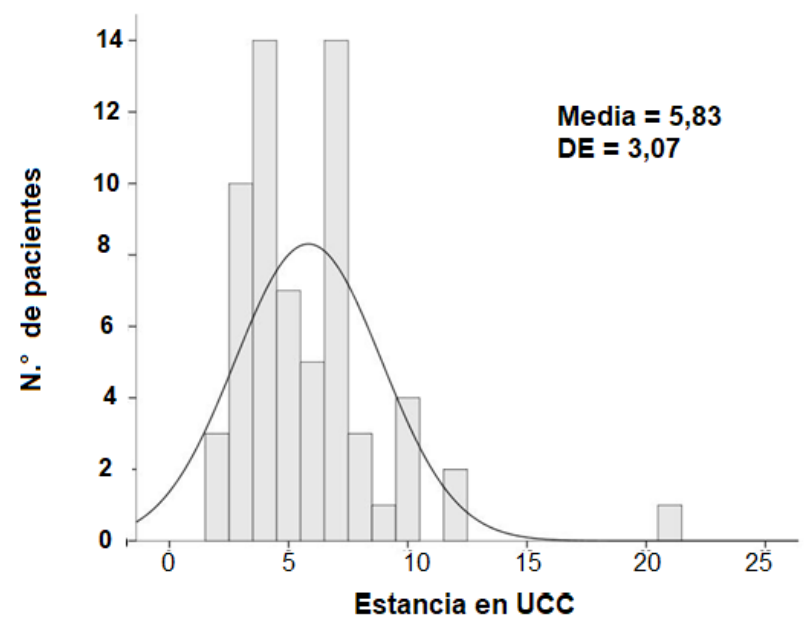

Figura 1. Distribución de estancia hospitalaria

En la tabla 2 se muestra la distribución de fracción de eyección ventricular izquierda y estancia hospitalaria para lo que se empleó OR. Se encontró que el $53 \%$ de los pacientes hospitalizados por insuficiencia cardiaca aguda presentan una fracción de eyección menor a $40 \%$.

Tabla 2. Frecuencia de fracción de eyección y estancia hospitalaria. Hospital Guillermo Almenara Irigoyen, 2015

\section{Fracción de eyección}

\section{Estancia hospitalaria}

Al calcular el OR entre la fracción de eyección del ventrículo izquierdo (mayor o igual a $40 \%$ / menor a $40 \%$ ) y el tiempo de estancia hospitalaria (mayor a cinco días o hasta cinco días) se encontró un valor de 1,016 (IC $95 \%$ : 0,380-2,717).

Análisis de correlación de las variables: relación Ele', diámetro de vena cava inferior $(\mathrm{VCl})$ y presión sistólica de arteria pulmonar (PSAP) con estancia hospitalaria

Se encontró que la media de la relación E/e' fue 17,3 y la
DE (desviación estándar) fue 9,2. La media del diámetro de vena cava inferior fue $18,02 \mathrm{~mm}$ y la DE 5,4. Se utilizó la correlación de Pearson para las variables relación E/e', diámetro de $\mathrm{VCl}$ y PSAP. El nivel de significación se fijó a dos colas ya que podría existir una asociación inversa a la planteada en el problema. En el caso de relación E/e' se realizó el análisis con 62 pacientes por ausencia de datos en dos pacientes. Para la relación $E / e^{\prime}$ se encontró $p=0,002$ y para el diámetro de $\mathrm{VCl}$ se encontró $p=0,006$ como se muestra en la tabla 3.

Tabla 3. Correlación entre relación E/e', diámetro de VCI, PSAP y estancia hospitalaria. Hospital Guillermo Almenara Irigoyen, 2015

\section{Variables correlacionadas}

\section{Estancia hospitalaria}

$$
\text { Pearson } \mathbf{R}
$$

N. ${ }^{\circ}$ pacientes

62

Relación E/e

0,391

0,43

0,23

64
64 
En relación a la presión arterial sistólica de la arteria pulmonar (PSAP), se encontró que la media fue de 48,2
mmHg y la DE 22,5. El $70 \%$ de los pacientes tenía PSAP > 35 $\mathrm{mmHg}$, tal como se muestra en la tabla 4.

Tabla 4. Frecuencia de presión sistólica de arteria pulmonar y estancia hospitalaria. Hospital Guillermo Almenara Irigoyen, 2015

\begin{tabular}{lccc}
$\begin{array}{c}\text { Presión sistólica } \\
\text { de arteria pulmonar }\end{array}$ & Hasta 5 días & Mayor 5 días & N. ${ }^{\circ}$ pacientes \\
\hline Menor a $35 \mathrm{mmHg}$ & 14 & 5 & $19(30 \%)$ \\
Mayor o igual a $35 \mathrm{mmHg}$ & 20 & 25 & $45(70 \%)$ \\
Total & 34 & 30 & $64(100 \%)$
\end{tabular}

Análisis del índice de colapso de vena cava inferior (VCI-IC) en relación a la estancia hospitalaria

En el caso de $\mathrm{VCl}-\mathrm{IC}$ se encontró que en la mitad de los casos había colapso mayor a 50 \% y en la otra mitad no había colapso, tal como se muestra en la tabla 5. Para establecer si existe asociación entre $\mathrm{VCl}-\mathrm{IC}<50 \%$ y estancia mayor a cinco días se encontró OR de 299,7 (IC $95 \%$ : 29 -3046,6) con un valor de $p<0,0000$.

Tabla 5. Frecuencia de VCI-IC y estancia hospitalaria. Hospital Guillermo Almenara Irigoyen, 2015

\begin{tabular}{lccc}
\multicolumn{1}{l}{ Variables } & Estancia mayor 5 dias & Estancia hasta 5 días & Total \\
\hline $\mathrm{VCl}-\mathrm{IC}<50 \%$ & $29(45,3 \%)$ & $3(4,7 \%)$ & $32(50 \%)$ \\
$\mathrm{VCl}-\mathrm{IC}>50 \%$ & $1(1,6 \%)$ & $31(48,4 \%)$ & $32(50 \%)$ \\
\hline Total & $30(47 \%)$ & $34(53 \%)$ & $64(100 \%)$
\end{tabular}

Nivel predictivo del diámetro de $\mathrm{VCl}$ y del valor Ele" en relación a estancia hospitalaria

Se realizó un análisis de regresión lineal entre la variable dependiente (estancia hospitalaria) y variable independiente (relación E/e') y se obtuvo un $\mathrm{R}^{2}=0,153$. De la misma manera, cuando se realizó entre estancia hospitalaria y diámetro de $\mathrm{VCl}$ se encontró $\mathrm{R}^{2}=0,149$ (Figuras 2 y 3 ).

Cuando se realiza el análisis de regresión lineal de ambas variables conjuntamente, se obtiene un $\mathrm{R}^{2}=0,28$.

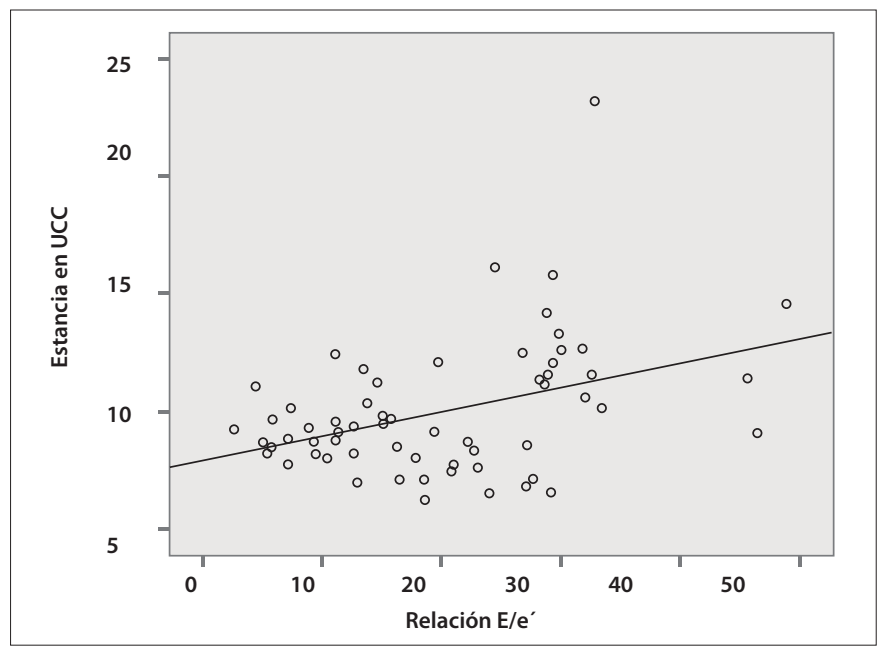

Figura 2. Gráfico de puntos del modelo de regresión parcial Variable dependiente: estancia hospitalaria 


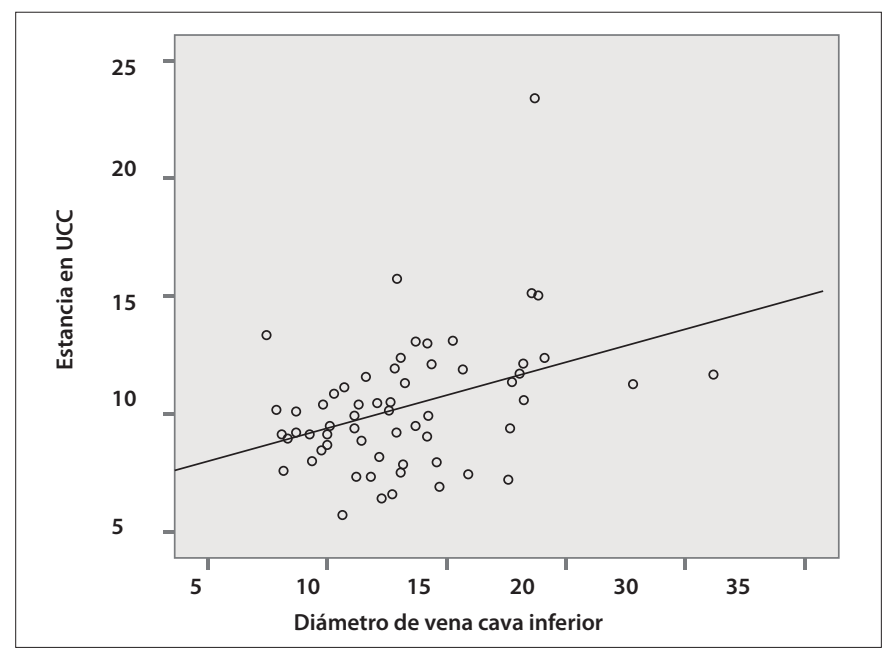

Figura 3. Gráfico de puntos del modelo de regresión parcial Variable dependiente: estancia hospitalaria

\section{DISCUSIÓN}

La insuficiencia cardiaca aguda representa un número importante de ingresos hospitalarios. La edad media y la proporción de varones en los estudios ADHERE (72 años, $48 \%$ ), OPTIMIZE-HF (73 años, $48 \%$ ) y EFHS II (70 años, $61 \%$ ) fueron similares ${ }^{(6)}$. En estos mismos estudios los antecedentes de enfermedad coronaria (60-70\%), hipertensión arterial $(70 \%)$, diabetes $(40 \%$ ) y fibrilación auricular (30-40\%) también fueron comparables.

Existen algunas diferencias con relación a esta investigación. En este trabajo, la edad media encontrada fue 67,8 años y la proporción de varones $61,5 \%$. El grupo etario con mayor prevalencia es el de los mayores de 75 años $(43,1 \%)$. Los varones son los más afectados $(61,5 \%)$ y hubo menor prevalencia de enfermedad coronaria $(35,4 \%)$.

En la insuficiencia cardiaca aguda, el mecanismo fisiopatológico fundamental es el incremento de la presión diastólica del ventrículo izquierdo que origina una congestión sistémica o pulmonar. Estos cambios ocasionan que luego de cada ingreso hospitalario por insuficiencia cardiaca aguda se produzca un compromiso estructural o funcional del miocardio y el siguiente evento agudo, probablemente, sea de mayor severidad (3). En consecuencia, nuestro reporta una asociación significativa entre el diagnóstico previo de insuficiencia cardiaca y la estancia hospitalaria mayor a cinco días, lo que incrementa cuatro veces la probabilidad de mantenerse hospitalizado por más de cinco días, OR de 4,16; $p=0,016$ (Tabla 1).

El promedio de estancia hospitalaria, según los diferentes registros, fue de 4 días (ADHERE y OPTIMIZE-HF), once días (EFHSI) y nueve días (EFHS II). De ellos, en el ADHERE el $23 \%$ ingresaron a una unidad de cuidados coronarios y el $51 \%$ en el EFHS II ${ }^{(1)}$. En este estudio solo se considera la estancia en unidad coronaria, que fue de 5,83 días.

En relación a la fracción de eyección del ventrículo izquierdo (FEVI) está demostrado su valor pronóstico a corto y largo plazo en los pacientes con insuficiencia cardiaca crónica. Sin embargo, esto no ocurre en los pacientes con insuficiencia cardiaca aguda porque alrededor del $50 \%$ tienen FEVI preservada. En nuestro trabajo no encontramos una correlación entre la FEVI y la estancia hospitalaria. Se encontró un OR entre $\mathrm{FEVI} \geq 40 \%$ y < a $40 \%$ y estancia hospitalaria mayor a cinco días o hasta cinco días de 1,016 (IC $95 \%$ : 0,380 - 2,717). Los resultados de Miro et al. fueron similares ${ }^{(16)}$.

Las variables relación E/e' y diámetro de vena cava inferior están asociadas al estado de volemia de los lados izquierdo y derecho del corazón, respectivamente ${ }^{(5,9,22,23)}$. Mientras mayor sea la sobrecarga de volumen, el paciente estará más comprometido y, por lo tanto, el tiempo de hospitalización, la mortalidad y las nuevas hospitalizaciones serán mayores ${ }^{\left({ }^{9}\right.}$.

En este estudio se encontró que el $58 \%$ de los pacientes con insuficiencia cardiaca aguda tenían un valor de $E / e^{\prime}$ mayor a 13. Se encontró que el valor de $E / e^{\prime}$ está asociado a la estancia hospitalaria; es decir, existe correlación entre una mayor estancia en unidad coronaria y un valor elevado E/e', por lo que se puede afirmar que el $39 \%$ de la mayor estancia de un paciente con insuficiencia cardiaca aguda se asocia a un mayor valor de E/e' y esta correlación es estadísticamente significativa $(p=0,002)$ (Tabla 3$)$. Se puede utilizar este parámetro como indicador de riesgo o severidad para tipificar a los pacientes que tendrán una estancia prolongada en unidad de cuidados coronarios. 
El diámetro de vena cava inferior es un parámetro ecocardiográfico que indica la sobrecarga de volumen en el lado derecho del corazón que suele ser una consecuencia de la sobrecarga en el lado izquierdo del corazón ${ }^{(24)}$. Se puede utilizar en el diagnóstico de insuficiencia cardiaca crónica y también en los casos de insuficiencia cardiaca aguda.

En este estudio, el $44 \%$ de los pacientes con insuficiencia cardiaca aguda tenían el diámetro de la vena cava inferior mayor a $21 \mathrm{~mm}$. Al analizar su correlación con la estancia hospitalaria se encontró que es significativa $(p=0,006)$. En términos numéricos, se puede decir que el $34 \%$ de la mayor estancia hospitalaria de un paciente con insuficiencia cardiaca aguda en la unidad coronaria se asocia a un mayor diámetro de vena cava inferior (Tabla 3).

El índice de colapso de vena cava inferior se emplea junto con el diámetro de vena cava inferior para determinar la presión de aurícula derecha, y es un indicador de sobrecarga de volumen. El índice de colapso se puede utilizar para el diagnóstico de insuficiencia cardiaca aguda.

En nuestro trabajo, la utilidad del índice de colapso no se encuentra en el diagnóstico de insuficiencia cardiaca aguda, puesto que se encontró un índice de colapso menor a $50 \%$ en la mitad de los pacientes. Este parámetro es útil al tipificar a los pacientes con estancia hospitalaria mayor a cinco días, puesto que hubo una asociación significativa $(p<0,0000)$ entre índice de colapso y estancia prolongada.

La hipertensión pulmonar, definida como presión arterial sistólica pulmonar mayor a $35 \mathrm{mmHg}$, es prevalente en la insuficiencia cardiaca crónica (medida por ecocardiografía) $\mathrm{y}$ es un predictor de nuevas hospitalizaciones y de mortalidad a largo plazo (13,24-26). En la insuficiencia cardiaca aguda, sistólica o diastólica también existe un incremento en los valores de presión arterial pulmonar por transmisión retrógrada de la presión en cavidades izquierdas.

Encontramos que en la mayoría de los pacientes (70 \%) la presión sistólica de arteria pulmonar es mayor a $35 \mathrm{mmHg}$ (Tabla 4). Sin embargo, al utilizar la correlación de Pearson entre el valor de presión sistólica de arteria pulmonar y estancia hospitalaria se encontró una correlación débil $(R=0,223)$ y estadísticamente no significativa $(p=0,76)$, lo que probablemente se debe al tamaño de la muestra (Tabla 3).

No es posible establecer un punto de corte específico por la cantidad de casos y los valores obtenidos para cada variable y su relación con cada paciente, aunque es evidente que el valor $\mathrm{E} / \mathrm{e}^{\prime}$ y el diámetro de la $\mathrm{VCl}$ se asocian con una mayor estancia hospitalaria. Sin embargo, se ha realizado el análisis de regresión, que establecerá un modelo predictivo sobre el comportamiento de las variables.
En el análisis de regresión lineal entre la relación E/e' y el diámetro de $\mathrm{VCl}$ con estancia hospitalaria en la unidad coronaria, cada variable explica, por sí sola, el $15,6 \%(p=0,001)$ del comportamiento de la estancia hospitalaria; mientras que las dos variables juntas pueden predecir el $28,2 \%(p=0,002)$ del comportamiento de la estancia hospitalaria. Se pudo obtener un efecto aditivo al combinar ambas variables y establecer un modelo predictivo debido a que el comportamiento es estadísticamente significativo. Existen pocos estudios que combinen estos índices ecocardiográficos.

En el análisis de regresión parcial también se encontró relación entre cada una de las variables de manera independiente: E/e' y estancia hospitalaria $\left(R^{2}=0,153\right)$ y entre diámetro de $\mathrm{VCl}$ y estancia hospitalaria $\left(R^{2}=0,149\right)$.

La relación que existe entre el aumento de E/e' y estancia hospitalaria puede explicarse por el incremento que existe en la volemia y en la presión de cavidades izquierdas que ocurre en los cuadros de insuficiencia cardiaca aguda. Esto origina que el valor de $E / e^{\prime}$ esté aumentado $\left(E / e^{\prime}>13\right)$ y el incremento de este parámetro se observó en aquellos pacientes que estaban más comprometidos, es decir, en los que tuvieron una estancia hospitalaria prolongada (mayor a cinco días) y, además, presentó una asociación significativa.

El aumento de presiones y volemia en las cavidades derechas transmitidas desde el lado izquierdo pueden ocasionar el incremento en el diámetro y el índice de colapso de vena cava inferior $\mathrm{a}<50 \%$. Este fenómeno ocurre en insuficiencia cardiaca aguda y es un marcador se severidad porque se asocia a la estancia hospitalaria prolongada.

Este estudio aporta datos útiles en situaciones de duda diagnóstica o para determinar el pronóstico a corto plazo en los casos de insuficiencia cardiaca aguda. Estos parámetros ecocardiográficos no son invasivos, son fáciles de obtener y de bajo costo.

Debido al diseño del estudio, las variables empleadas y el tamaño de la muestra no es posible establecer un valor específico de E/e' o diámetro de vena cava inferior que sea útil como punto de corte para discriminar entre una estancia menor a cinco días o mayor a cinco días. Solo se pueden establecer asociaciones entre variables. Para establecer un punto de corte específico se requiere de trabajos con un mayor número de casos.

Se concluye que el incremento en la relación E/e', el mayor diámetro de vena cava inferior y el índice de colapso de vena cava inferior $<50 \%$ están asociados significativamente a una estancia hospitalaria prolongada en pacientes con insuficiencia cardiaca aguda en la unidad de cuidados coronarios, lo que no sucede con la presión sistólica de arteria pulmonar. 
Contribuciones de autoría: Ciro Barrantes participó de la concepción del trabajo de investigación, diseño, recolección de datos, análisis, interpretación de datos, revisión crítica y redacción del artículo.

Fuentes de financiamiento: El artículo ha sido financiado por el autor.

Conflicto de interés: El autor declara no tener ningún conflicto de interés.

\section{REFERENCIAS BIBLIOGRÁFICAS}

1. Farmakis D, Parissis J, Lekakis J, Filippatos G. Insuficiencia cardiaca aguda: epidemiología, factores de riesgo y prevención. Rev Esp Cardiol. 2015; 68(3): 245-8.

2. Llorens P, Escoda R, Miró 0, Herrero-Puente P, Martín-Sánchez FJ, Jacob J, et al. Características clínicas, terapéuticas y evolutivas de los pacientes con insuficiencia cardiaca aguda atendidos en servicios de urgencias españoles: registro EAHFE (Epidemiology of acute heart failure in spanish emergency departments). Emergencias. 2015; 27: 11-22.

3. Bonow R, Mann D, Zipies D, Libby P, Braunwald E. Tratado de Cardiología de Braunwald. Texto de medicina cardiovascular. 9a ed. Barcelona: Elsevier España; 2013.

4. Ricci JE, Kalmanovich E, Robert C, Chevallier T, Aguilhon S, Solecki $\mathrm{K}$, et al. Manejo de insuficiencia cardiaca aguda: contribución de la evaluación ecocardiográfica diaria en el ajuste de la terapia con medida de impacto en la tasa de readmisiones a 30 días (JECICA). Contemp Clin Trials Commun. 2018; 9(12): 102-8.

5. Persson H, Donal E, Lund L, Matan D, Oger E, Hage C, et al. Importancia de enfermedad cardiaca estructural y disfunción diastólica en insuficiencia cardiaca con fracción de eyección preservada según las guías ESC: un subestudio en el estudio KaRen. Int J Cardiol. 2019; 274: 202-7.

6. Arques $\mathrm{S}$, Roux $\mathrm{E}$. Utilidad de test de péptidos natriuréticos y ecocardiograma doppler al lado del paciente en el diagnóstico de insuficiencia cardíaca aguda. Ann Cardiol Angeiol. 2016; 65(5): 334-9.

7. Papadimitriou L, Georgiopoulou V, Kort S, Butler J, Kalogeropoulos A. Ecocardiografía en insuficiencia cardiaca aguda: perspectivas actuales. J Cardiac Fail. 2016; 22: 82-94.

8. Donal E, Lund L, Oger E, Hage C, Persson H, Reynaud A. Nuevos predictores ecocardiográficos de resultados clínicos en pacientes que se presentan con insuficiencia cardiaca y fracción de eyección preservada: subanálisis del estudio KaRen. Eur J Heart Fail. 2015; 17: 680-8.

9. Soltani MH, Jamshir M, Taghavi S, Golpira R, Nasiri M, Amin A, et al. Predictores ecocardiográficos de empeoramiento de función renal en insuficiencia cardiaca aguda: observaciones del registro RASHF. ESC Heart Failure. 2018.

10. Tchernodrinski S, Lucas BP, Athavale A, Candotti C, Margeta B, Katz A. Cambios en el diámetro de vena cava inferior después de furosemida intravenosa en pacientes diagnosticados con insuficiencia cardiaca aguda descompensada. J Clin Ultrasound. 2015; 43(3): 187-93.

11. Badagliacca R, Ghio S, Correale M, Poscioa R, Camporotondo R, Ferraretti A. Significado pronóstico de la estimación ecocardiográfica de hipertensión pulmonary y de disfunción ventricular derecho en insuficiencia cardiaca aguda descompensada. Un estudio piloto en pacientes con insuficiencia cardiaca con fracción de eyección reducida. Int J Cardiol. 2018; 271: 301-5.

12. Matushita K, Sakata K, Satoh T, Yoshino H. Presión sistólica de arteria pulmonar y mortalidad en pacientes ancianos con falla cardiaca. J Am Geriatr Soc. 2019; 67(2): 323-8.

13. Mancuso F, Moisés V, Almeida D, Poyares D, Storti L, Brito F. Valor pronóstico de Eco 3D comparado a eco bidimensional en pacientes con insuficiencia cardiaca sistólica. Int J Cardiovasc Imaging. 2018; 34(4): 553-60.

14. Santas E, de la Espriella J, Mollar A, Valero E, Miñana G, Sanchis J. Estimación ecocardiográfica de la presión de arteria pulmonar y rehospitalización en pacientes con insuficiencia cardiaca. Int J Cardiol. 2017; 241: 407-10.

15. Donal E, Lund LH, Oger E, Bosseau C, Reynaud A. Importancia del tamaño de aurícula izquierda y presión pulmonar estimada combinadas para resultado clínico en pacientes que se presentan con insuficiencia cardiaca fracción de eyección preservada. Eur Heart J Cardiovasc Imaging. 2017; 18(6): 629-35.

16. Miró 0 , Javaloyes P, Gil V, Martín-Sánchez F, Jacob J, Herrero P, et al. Análisis comparativo de resultados a corto plazo de pacientes con insuficiencia cardiaca con fracción de eyección en rango medio después de descompensación aguda. Am J Cardiol. 2019; 123(1): 84-92.

17. Nagueh S, Smiseth 0 , Appleton C, Byrd B, Dokainish H, Edvardsen T. Recomendaciones para la evaluación de función diastólica ventricular izquierda por ecocardiografía: actualización de la Sociedad Americana de Ecocardiografía y la Asociación Europea de Imagen Cardiovascular. J Am Soc Echocardiogr. 2016; 29: 277-314.

18. Porter TR, Shillcutt SK, Adams MS. Guidelines for the use of echocardiography as a monitor for therapeutic intervention in adults: a report from the American Society of Echocardiography. J Am Soc Echocardiogr. 2015; 28: 40-56.

19. Lang R, Badano L, Mor-Avi V, Afilalo J, Armstrong A, Ernande L. Recomendaciones para cuantificación de cámaras cardiacas en adultos por ecocardiografía: actualización de la Sociedad Americana de Ecocardiografía y la Asociación Europea de Imagen Cardiovascular. J Am Soc Echocardiogr. 2015; 28: 1-39.

20. Galiè N, Humbert M, Vachiery JL, Gibbs S, Lang I, Torbicki A. Guía ESC/ERS 2015 sobre diagnóstico y tratamiento de la hipertensión pulmonar. Rev Esp Cardiol. 2016; 69(2): 177.e1-62.

21. Ponikowski P, Voors A, Anker S, Bueno H, Cleland J, Coats A. Guía ESC 2016 sobre el diagnóstico y tratamiento de la insuficiencia cardiaca aguda y crónica. Rev Esp Cardiol. 2016; 69(12): 1167.e1-85.

22. Tachjian A, Sanghai S, Stencel J, Parker M, Kakouros N, Aurigemma G. Estimación de la presión de aurícula izquierda en pacientes con síndrome coronario agudo: un estudio doppler ecocardiográfico y cateterismo cardiaco. J Am Soc Echocardiogr. 2019; 32(3): 365-74.

23. Nagueh SF, Smiseth O, Dokainish H, Andersen O, Abudiab M, Schutt $\mathrm{R}$, et al. Presión en aurícula derecha para estimación de la presión de llenado ventricular izquierda en pacientes con fracción de eyección ventricular izquierda normal: validación invasiva y no invasiva. J Am Soc Echocardiogr. 2018; 31(7): 799-806.

24. Ohman J, Harjola VP, Karjalainen P, Lassus J. Evaluación de la respuesta temprana por ultrasonido cardiotorácico rápido en insuficiencia cardiaca aguda: presiones de llenado cardiaco, congestión pulmonar y mortalidad. Eur Heart J Acute Cardiovasc Care. 2018; 7(4): 311-20.

25. Kalogeropoulos AP, Siwamogsatham S, Hayek S, Li S, Deka A, Marti $\mathrm{CN}$, et al. Evaluación ecocardiográfica de la presión sistólica de arteria pulmonar y resultados en pacientes ambulatorios con insuficiencia cardiaca. J Am Heart Assoc. 2014; 3(1): e000363.

26. Dzudie A, Kengne AP, Thienemann F, Sliwa K. Predictores de hospitalizaciones por insuficiencia cardiaca y mortalidad en pacientes con hipertensión pulmonar asociada con enfermedad cardiaca izquierda: una revisión sistemática. BMJ Open. 2014; 4(7): e004843. 


\section{Correspondencia:}

Ciro Barrantes

Dirección: Avenida Grau 800 La Victoria. Lima, Perú.

Celular: 941392086

Correo electrónico: cirobarrantes@yahoo.com

\section{Recibido: 20 de enero de 2020}

Evaluado: 16 de abril de 2020

Aprobado: 16 de julio de 2020

(c) La revista. Publicado por Universidad de San Martín de Porres, Perú.

(c) Br Licencia de Creative Commons Artículo en acceso abierto bajo términos de Licencia Creative Commons Atribución 4.0 Internacional. (http://creativecommons.org/licenses/by/4.0/)

ORCID iDs

Ciro Barrantes

(ㄱ) https: / / orcid.org/0000-0003-3126-6226 